\title{
Caring for patients with opioid use disorder in the hospital
}

\author{
Joseph H. Donroe MD, Stephen R. Holt MD MS, Jeanette M. Tetrault MD
}

Author interview at https://soundcloud.com/cmajpodcasts/160290-view

\begin{abstract}
Competing interests: Jeanette Tetrault has received consultancy fees from Cardiocore, for work outside the scope of the current article. No other competing interests were declared.
\end{abstract}

This article has been peer reviewed.

Correspondence to: Joseph Donroe, joseph. donroe@yale.edu

CMAJ 2016. DOI:10.1503 /cmaj.160290
$\mathrm{O}$ pioid use disorder refers to a problematic pattern of opioid use leading to clinically significant impairment or distress, as defined by the Diagnostic and Statistical Manual of Mental Disorders, fifth edition (DSM-5). ${ }^{1}$ The term "opioid" refers to any substance that acts at opioid receptors, including prescription medications, such as morphine, and illicit drugs, such as heroin. All practitioners, regardless of specialty, will care for patients with this prevalent, chronic medical condition, including during acute hospital admissions.

Patients with substance use disorders are among the highest users of health care, incurring disproportionately high health care costs and frequently requiring readmission to hospital. ${ }^{2-4}$ Nonetheless, a recent retrospective study concluded that addiction-specific interventions remain underused, both during acute hospital admissions and at the time of discharge. ${ }^{5}$

In this review, we consider issues unique to adult patients with opioid use disorder during acute hospital admissions. We use current guidelines, systematic reviews and current studies to familiarize readers with the use of opioid agonist therapy (Box 1), ${ }^{6-14}$ management of opioid withdrawal and overdose, acute pain management and safe discharge practices for hospital inpatients with opioid use disorder (Box 2).

\section{- Ker poInTS}

- Physicians from many specialties may care for patients with opioid use disorder in the inpatient setting.

- An acute hospital admission is an opportunity to engage patients with this common chronic condition in addiction treatment and to affect the course of their illness.

- Acute pain can be frustrating for patients with opioid use disorder and their health care providers, although an informed approach can improve outcomes.

- Before discharge from hospital, providers should discuss long-term addiction treatment options and harm reduction strategies, screen for infectious diseases and immunization status, and prescribe opioid medications, in limited amounts, when indicated for pain.

\section{How can patients with opioid use disorder be identified in hospital?}

Substance use disorders are common in the hospital setting. In two studies conducted in urban hospitals in the United States, substance use not related to alcohol or tobacco among hospital inpatients was about $11 \%$, not including patients stabilized on opioid agonist therapy. ${ }^{15,16}$ In practice, either inpatients will have a previous diagnosis of opioid use disorder or providers will become concerned about this condition in patients who exhibit certain "red flag" behaviours. These behaviours may be evident only after discussions with primary care providers and upon review of data from prior hospital admissions and prescription monitoring programs. They include injecting oral formulations of pain medications, using other illicit drugs concurrently, having multiple opioid prescribers, being inflexible with pain management plans and having recurrent admissions for pain without identifiable cause. ${ }^{17}$ Once opioid use disorder is suspected, the DSM-5 criteria ${ }^{1}$ can be used to establish a diagnosis and determine severity.

\section{How is opioid withdrawal managed?}

Opioid withdrawal may occur if opioids are stopped abruptly or the dose is substantially reduced. For patients taking short-acting opioids, withdrawal symptoms (Table 1$)^{18}$ may begin $6-12$ hours after the last dose, peak in 36-72 hours and last for 7-10 days. With longer-acting opioids, symptoms may begin 24-48 hours after the last dose, peak at 72-96 hours and last for up to several weeks. ${ }^{18,19}$ The severity of opioid withdrawal can be graded with validated instruments such as the Clinical Opioid Withdrawal Scale, the Subjective Opioid Withdrawal Scale and the Objective Opioid Withdrawal Scale. ${ }^{18-20}$ Withdrawal from other 
substances, particularly alcohol and benzodiazepines, can be life-threatening, and vigilance for detecting such withdrawal is therefore important.

The goals of treating withdrawal are to alleviate unnecessary distress, maintain the therapeutic alliance between patient and provider, facilitate treatment of the primary reason for admission and increase the patient's engagement in longterm addiction management. According to a 2010 systematic review, ${ }^{21}$ there was no statistically significant difference in completion of opioid detoxification between the opioid agonists buprenorphine and methadone (odds ratio 1.64, 95\% confidence interval [CI] 0.68-3.79). As such, choosing between them should take into account the patient's preference and commitment to longterm addiction treatment, comorbid medical conditions, potential adverse effects (e.g., QTc prolongation), medication interactions (e.g., with some antiretroviral medications) and availability of outpatient providers able to continue the opioid agonist therapy. ${ }^{21}$ Methadone initiated at 10
$30 \mathrm{mg}$ daily and slowly titrated to a total daily dose of $20-40 \mathrm{mg}$ is usually sufficient to treat withdrawal symptoms. ${ }^{18,20}$ Alternatively, buprenorphine may be initiated, according to standard protocols, once moderate opioid withdrawal is evident (Figure 1).22 Buprenorphine initiated too early can precipitate withdrawal.

Continuation of opioid agonist therapy after discharge, for long-term management of opioid use disorder, is preferred. However, if such therapy will not be continued beyond the hospital stay (because of patient preference or lack of available outpatient providers), consensus guidelines recommend tapering off over three to five days, with a daily dose reduction of 5-10 mg (methadone) or $2 \mathrm{mg}$ (buprenorphine), although longer tapering schedules are preferred when possible. ${ }^{18,22}$

According to a 2016 Cochrane review, ${ }^{23}$ $\alpha_{2}$ adrenergic agonists (clonidine and lofexidine) are more effective than placebo for prevention of severe withdrawal (relative risk [RR] $0.32,95 \%$ CI $0.18-0.57$ ) and for completion of

\section{Box 1: Management of opioid use disorder}

\section{Opioid agonist therapy}

Therapy with opioid agonists, including methadone, buprenorphine and diacetylmorphine, can be used for long-term treatment of opioid use disorder and for acute management of withdrawal symptoms.

Methadone is a synthetic opioid agonist with a variable half-life, averaging 24-36 hours at steady state. ${ }^{6}$ This characteristic makes once-daily dosing effective for treating opioid use disorder and withdrawal, but it also increases the risk of overdose if the dose is escalated too quickly, because of accumulation of drug in the serum. It is available as a liquid or tablet, although when used for addiction the liquid formulation is preferred. Among inpatients, relevant adverse effects may include constipation, drowsiness and QT interval prolongation.7

Buprenorphine is a semisynthetic partial agonist with very high affinity at the opioid $\mu$ receptor. Its partial agonist property confers a ceiling effect on analgesia and respiratory depression, although 2 autopsy studies highlighted the risk of overdose when other respiratory depressants are used concomitantly. 8,9 For the treatment of opioid use disorder, the most commonly used formulation of buprenorphine is the combination product, buprenorphine-naloxone, available as a dissolvable sublingual tablet or film. Buprenorphine without naloxone is available in the United States; in Canada, it can be prescribed in specific clinical situations, such as pregnancy, through Health Canada's Special Access Programme. Naloxone is clinically active only if injected, and it is therefore added to the combination product as a deterrent to intravenous administration of the oral formulation. Common adverse effects relevant to hospital inpatients include constipation and headache, and transaminases may become elevated in patients with chronic hepatitis $\mathrm{C}$ or alcohol use. ${ }^{10}$

Both methadone and buprenorphine are considered safe for patients with renal disease, although dose reductions may be needed in those with glomerular filtration rate below $10 \mathrm{~mL} / \mathrm{min} .{ }^{11}$ Likewise, both are primarily metabolized in the liver, so administration to patients with severe liver impairment requires close monitoring. In Canada, the prescription of methadone for the treatment of opioid use disorder requires an exemption under section 56 of the Controlled Drugs and Substances Act, although a temporary exemption can be obtained when caring for a patient with opioid use disorder during an acute hospital admission. ${ }^{12}$ Restrictions regarding buprenorphine prescribing vary by province, and providers should refer to local regulations. ${ }^{13}$

Diacetylmorphine (the active ingredient in heroin) is a short-acting, semisynthetic injectable opioid agonist that has received increasing attention for treatment of severe opioid use disorder in patients with failure of more traditional approaches involving oral agonists. ${ }^{14}$ This drug is not available for the treatment of opioid use disorder in the US, and it has not yet been studied as a medication for inpatients with opioid use disorder.

\section{Opioid antagonist therapy}

Treatment options for opioid use disorder in the outpatient setting also include the opioid antagonist naltrexone. With its high binding affinity and long half-life, naltrexone prevents the analgesic and euphoric effects of opioid agonists, thereby leading to a reduction in the use of all opioids. ${ }^{10}$ Importantly, if a patient takes the first dose of naltrexone while there are still active opioids in the circulation, the naltrexone may abruptly precipitate withdrawal. Naltrexone is available in 2 formulations: oral ( $50 \mathrm{mg}$ taken once daily) and intramuscular (380 mg monthly). Common adverse effects of both forms include nausea, which typically resolves after a few days of use, and hepatotoxicity, which is rare; in addition, with the intramuscular form, an idiosyncratic injection site reaction may occur, lasting for several weeks. Patients receiving maintenance naltrexone therapy who are admitted with acute pain may require very high doses of opioids to override the blocking effects of naltrexone, depending upon the timing of the most recent dose. ${ }^{10}$ 
treatment (RR 1.95, 95\% CI 1.34-2.84). Of note, although $\alpha_{2}$ adrenergic agonists improve withdrawal symptoms, they do not address craving. Other adjunct medications targeting withdrawal symptoms are listed in Table 1.

\section{How is opioid overdose managed?}

Opioid overdose in the inpatient setting may occur when opioid tolerance is overestimated (i.e., following a period of abstinence), when opioids are aggressively dosed or administered to patients with new renal or hepatic impairment, when medications that influence opioid metabolism or potentiate respiratory depression are coadministered and when patients undertake self-administration of opioids..$^{24}$ Overdose should be suspected in patients with any combination of depressed mental status, decreased respiratory rate or chest wall rise, and miotic pupils. ${ }^{25}$

Assessment begins with basic life support and includes a review of all administered medications. A focused examination includes inspection of the skin for transdermal opioid patches. Patients who are protecting their airway and who have good oxygen saturation levels, a respiratory rate greater than 10-12 breaths/min and no evidence of hypercarbia by blood gas or capnography can simply be observed in a monitored setting. ${ }^{25}$

For patients with apnea, bag-valve mask ventilation should be performed and naloxone administered by the intravenous route. ${ }^{25}$ Naloxone can also be administered intramuscularly, intranasally or via endotracheal tube, depending on the clinical scenario. The effects of naloxone last 20-90 minutes; therefore, suspected overdoses involving long-acting opioids may require repeat dosing or intravenous infusion. If the air- way is difficult to maintain or the respiratory rate does not improve after escalating doses of naloxone, orotracheal intubation should be performed. Patients with recurrent apnea and those who require a naloxone infusion or intubation should be transferred to an intensive care unit. ${ }^{25}$

\section{How should acute pain be managed in opioid use disorder?}

Acute pain is common among hospital inpatients, and early recognition and management alleviates the patient's discomfort while facilitating management of other medical issues. ${ }^{26,27} \mathrm{In}$

Box 2: Evidence used in this review
For each clinical question included in this
review, a National Center for Biotechnology
Information "PubMed" and "Books" database
search was performed, with search terms
depending on the clinical question. For
example, for the section "How is opioid
withdrawal managed?" the search terms were
"opioid withdrawal," "methadone,"
"buprenorphine" and "alpha adrenergic."
Searches were limited to full-text English-
language articles published after the year 2000.
Additionally, we reviewed reference lists from
guideline statements and seminal review
articles. We chose the most relevant and recent
articles presenting results from randomized
controlled trials, case-control and cohort
studies, meta-analyses, guideline statements
and reviews. We included some case series
when the available literature was limited. We
reviewed 186 citations, of which 56 contributed
to the data included in this review. The lead
author (J.H.D.) reviewed all of the articles, and
the coauthors (S.R.H. and J.M.T.) reviewed
selected articles.

Table 1: Signs and symptoms of opioid withdrawal and targeted management ${ }^{18}$

\begin{tabular}{|c|c|}
\hline Sign or symptom* & Pharmacologic management \\
\hline $\begin{array}{l}\text { Tachycardia, hypertension, hyperthermia, } \\
\text { diaphoresis, lacrimation, rhinorrhea, } \\
\text { piloerection, mydriasis, yawning }\end{array}$ & $\alpha_{2}$ agonist (clonidine, lofexidine)* \\
\hline Anxiety, insomnia & $\begin{array}{l}\text { Antihistamine (e.g., diphenhydramine, hydroxyzine), } \\
\text { sedating antidepressant (e.g., trazodone) }\end{array}$ \\
\hline Myalgia, bone pain & $\begin{array}{l}\text { Acetaminophen, nonsteroidal anti-inflammatory drugs } \\
\text { (e.g., ibuprofen) }\end{array}$ \\
\hline Abdominal cramps & Antispasmodic (e.g., dicyclomine) \\
\hline Nausea and vomiting & $\begin{array}{l}\text { Antiemetic (e.g., prochlorperazine, promethazine, } \\
\text { ondansetron) }\end{array}$ \\
\hline Diarrhea & Antidiarrheal (e.g., loperamide) \\
\hline Hyperreflexia & No specific therapy \\
\hline
\end{tabular}


addition, studies have identified associations between the undertreatment of pain in individuals with opioid use disorder and illicit opioid use by inpatients and discharges against medical advice. $^{28,29}$

Basic pain management principles apply, including assessing the pain with standardized assessment tools, addressing the underlying cause, maximizing the use of physical measures (ice, heat, massage, bracing) and administering non-opioid medications (nonsteroidal antiinflammatory drugs. acetaminophen, nerve blocks). ${ }^{26,30}$ When opioid analgesics are needed, prescription drug monitoring programs should be consulted where available. ${ }^{31}$ Because intermittent intravenous administration of opioids is

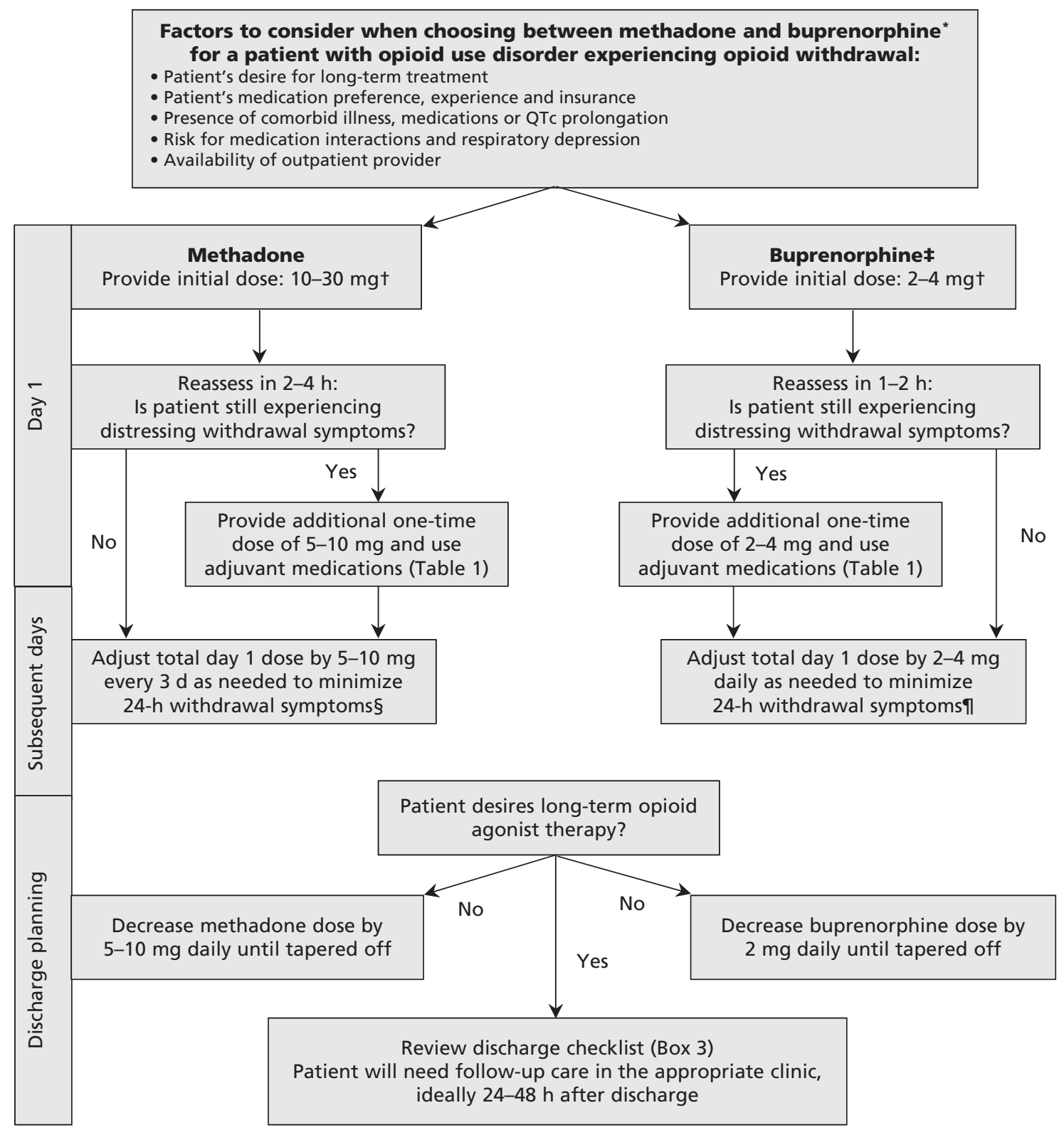

Figure 1: Initiation of methadone or buprenorphine for the treatment of withdrawal in the inpatient setting. ${ }^{18,22} *$ Long-term opioid agonist therapy is preferred and should be encouraged. Methadone should be used with caution in patients with QTc prolongation. +Consider the lower end of the dose range for older adults, adolescents, patients who are using other central nervous system depressants, those otherwise at higher risk for respiratory depression and those whose opioid use is infrequent. Higher initial doses may be needed for patients with higher daily opioid use. ¥Buprenorphine may be administered once a patient is in moderate withdrawal (Clinical Opioid Withdrawal Scale > 12). A combination buprenorphine-naloxone product is available in Canada, and buprenorphine alone can be obtained through Health Canada's Special Access Programme if necessary. §Methadone doses above $\mathbf{4 0} \mathbf{~ m g}$ are rarely needed to treat withdrawal. ๆlt is recommended to not exceed buprenorphine $8 \mathrm{mg}$ on day 1 and $16 \mathrm{mg}$ on day 2. 
unnecessarily reinforcing, a transition from intravenous to oral opioids should occur once the patient can tolerate oral medications and the pain is reasonably controlled, typically within 24 hours after admission. ${ }^{32}$ When possible, medications that are currently or were formerly problematic for the patient should be avoided. ${ }^{33}$ Aspects of acute pain management unique to patients with opioid use disorder are reviewed in the following sections.

\section{Provider and patient biases}

Interviews with providers have commonly identified concerns about deception and manipulation on the part of patients with opioid use disorder who report pain. ${ }^{34,35}$ At the same time, patients with opioid use disorder fear that they will be labelled as "drug seekers," that their pain will go undertreated, that the underlying condition will go undiagnosed or that their opioid agonist therapy will be discontinued. ${ }^{34,36}$ Elements of an effective initial pain encounter include reviewing both patient and provider expectations regarding pain management, acknowledging prior difficult interactions, reassuring the patient that the pain will be addressed and that opioid agonist therapy will be continued or substituted if necessary, and reviewing the medication schedule. Involving the patient in decisions about pain management is particularly relevant for those with opioid use disorder and may prevent some of the stressful doctor-patient interactions related to opioids that both parties dislike. ${ }^{26,27,37}$

\section{Patients actively abusing opioids}

In light of the well-established phenomena of tolerance and opioid-induced hyperalgesia among chronic, active opioid users, patients with opioid use disorder will likely require higher doses of opioid than will non-opioiddependent patients. ${ }^{26,30,38}$ In patients with moderate to severe acute pain who currently use illicit opioids, the aim of management is to provide adequate analgesia and prevent opioid withdrawal. ${ }^{30}$

One strategy is to initiate methadone as outlined above and to prescribe additional shortacting opioids titrated to pain. ${ }^{39}$ This approach has the advantage of treating both the opioid use disorder and the pain. Alternatively, short-acting opioids can be used alone; however, physicians should be aware of the potential for withdrawal symptoms to occur between intermittent doses of short-acting agents if given too infrequently. The administration frequency of short-acting opioids generally should be scheduled, rather than on an "as needed basis," with hold parameters if the patient appears oversedated. ${ }^{26,27,30,37,38}$
Patients receiving opioid agonist therapy

Patients experiencing acute pain who are already receiving methadone maintenance therapy should continue to receive their usual dose, once confirmed by the outpatient treatment centre. The analgesic effects of methadone are much shorter than its effects on withdrawal and cravings. ${ }^{26,37}$ As such, if non-opioid modalities fail to provide sufficient relief, short-acting opioids should be added to methadone maintenance therapy to treat moderate to severe acute pain. It is difficult to predict the effect of the additional analgesics, and frequent reevaluation and dose titration are necessary. 26,30 In the setting of acute pain, the physician could consider splitting the patient's daily methadone dose into two or three times daily dosing, given the shorter analgesic effect of methadone; however, there is scant literature to support this approach. ${ }^{40}$

The analgesic effect of buprenorphine is also shorter than its effects on withdrawal and craving. Given its high affinity for the opioid $\mu$ receptor, buprenorphine effectively blocks the actions of most other opioids, thereby complicating acute pain management. ${ }^{41}$ Several strategies have been described to overcome this problem, predominantly based in expert opinion. The first is to continue daily buprenorphine and add short-acting opioids titrated to pain control. High-dose opioids, and possibly patient-controlled analgesia, will likely be needed. ${ }^{26,39,41-43}$ A second strategy uses the inherent analgesic properties of buprenorphine by giving the total daily dose divided three or four times daily. ${ }^{26,42}$ Doses of 4-8 mg every six to eight hours have been used to treat moderate to severe pain. ${ }^{41,43} \mathrm{~A}$ third strategy is to discontinue buprenorphine and use short-acting opioids to treat the acute pain. The blocking effects of buprenorphine wear off over 24-72 hours, so the patient must be carefully monitored for signs of overdose, given that the initial opioid dose will be much higher than ultimately needed. ${ }^{22}$ After the need for acute pain management has resolved, buprenorphine can be reintroduced.

\section{Is there benefit to inpatient initiation of opioid agonist therapy?}

Initiation of opioid agonist therapy during an acute hospital admission can facilitate management of other medical issues by relieving symptoms associated with withdrawal, reducing the rates of discharge against medical advice and 
increasing the likelihood that patients with opioid use disorder will transition to long-term outpatient addiction treatment. This approach, in turn, has been shown to reduce illicit opioid use, increase retention in addiction treatment, lower the risk of opioid-associated health problems such as HIV infection, decrease hospital utilization, improve psychosocial functioning and improve management of other comorbid medical and psychologic conditions. ${ }^{10,44}$

Several retrospective studies have identified patients with substance use disorders as having a high risk of discharge against medical advice, which is associated with increases in hospital readmissions and health care costs, as well as in the risk of death, highlighting the importance of prevention. ${ }^{45-48}$ Qualitative research and one systematic review found that opioid withdrawal, inadequate pain management, younger age, daily heroin injection and unstable employment were factors contributing to discharges against medical advice among patients with substance use disorders. ${ }^{29,49,50}$

Available evidence suggests that opioid agonist therapy, when paired with referral to outpatient addiction services, may reduce discharge against medical advice and increase engagement in postdischarge addiction treatment. ${ }^{51-55}$ One observational study of inpatients with opioid use disorder showed that initiation of methadone during the hospital stay, coupled with linkage to an outpatient addiction program, led to a high rate $(82 \%)$ of postdischarge follow-up. ${ }^{54} \mathrm{~A}$ randomized controlled trial showed that inpatient initiation of buprenorphine and referral to an outpatient addiction provider led to significantly better rates of long-term treatment for opioid use disorder than inpatient detoxification alone $(16.7 \%$ v. $3 \%, p=0.007$, at 6 months after the hospital stay)..$^{53}$

\section{What factors should be considered before discharge from hospital?}

Discharging patients with opioid use disorder requires advance planning, and providers should refer to the discharge checklist provided in Box 3. According to expert opinion, physicians should discuss overdose prevention with all patients who have opioid use disorder and should consider prescribing naloxone rescue medication. Patients should be reminded that after a period of

\section{Box 3: Discharge checklist*}

- Screened for associated infectious diseases during this hospital admission or previously? - HIV

- Hepatitis B, hepatitis C

- Screened for other substance use disorders?

- Addressed psychosocial comorbidities such as homelessness, depression, post-traumatic stress disorder?

- Immunizations up to date?

- Tetanus

- Hepatitis A, hepatitis B

- Influenza

- Pneumococcus

- Considered education and/or referral for harm reduction strategies?

- Needle exchange programs and supervised injection facilities (where available)

- Safe injecting practices

- Opioid overdose education and prescription of naloxone rescue doses

- Safe opioid storage, particularly if children living in the home

- Considered long-term treatment for opioid use disorder?

- Discussed initiation of opioid agonist therapy

- Referral to specialized addiction treatment centre

- Referral to pain specialist if indicated

- Referral for counselling interventions (i.e., self-help, mutual help, one-to-one counselling)

- Established primary care physician

- Thoughtful prescribing of new medications?

- Consideration of medication interactions if taking opioid agonist treatment

- If needed, short-term opioid analgesia with close follow-up after discharge

*Checklist developed by the authors. 
abstinence, such as a hospital stay, the risk of overdose is high because of loss of tolerance.

When patients with opioid use disorder have acute pain needs extending beyond the period of the admission, careful attention must be given to the duration of treatment and the frequency of administration of opioids, because of the risk of addiction relapse. ${ }^{56}$ In accordance with the guidelines for prescribing opioid treatment for chronic noncancer pain published recently by the US Centers for Disease Control and Prevention, physicians should prescribe the lowest effective dose and limit the quantity of opioids prescribed to the expected duration of severe acute pain, usually less than one week. ${ }^{31}$ Increased frequency of follow-up appointments to assess ongoing need for pain medication may be necessary, particularly when severe pain continues for longer than initially expected. ${ }^{31}$

\section{Conclusion}

The inpatient care of patients with opioid use disorder can be medically and psychosocially complex. In this review, we have highlighted the unique aspects of caring for this population during an acute hospital admission. Future studies should focus on strategies to reduce discharges against medical advice, to manage acute pain (particularly for patients receiving buprenorphine) and to increase engagement in long-term outpatient care (Box 4). When possible, we recommend seeking consultation from an addiction specialist or an inpatient addiction consult service, although this review provides a framework for other physicians to improve their care of inpatients with opioid use disorder.

At the core is the concept that addiction is a chronic disease, and thoughtful consideration of the underlying opioid use disorder should be part of the patient's daily care plan. Failure to engage with patients on their opioid use, offer appropriate treatments and create a thoughtful discharge plan represent a missed opportunity to affect the

\section{Box 4: Unanswered questions}

- What are the optimal strategies for successful transition of hospital inpatients with opioid use disorder into long-term outpatient treatment programs?

- What are optimal non-opioid strategies for managing pain in patients with opioid use disorder?

- What is the optimal strategy for managing acute, moderate to severe pain for patients who are receiving buprenorphine?

- What are the best strategies to mitigate against discharges against medical advice among patients with opioid use disorder? course of this common chronic condition and to prevent related morbidity and mortality.

\section{References}

1. American Psychiatric Association. Diagnostic and statistical manual of mental disorders. 5th ed. Arlington (VA): American Psychiatric Association Publishing; 2013.

2. Walley AY, Paasche-Orlow M, Lee EC, et al. Acute care hospital utilization among medical inpatients discharged with a substance use disorder diagnosis. J Addict Med 2012;6:50-6.

3. Neighbors CJ, Sun Y, Yerneni R, et al. Medicaid care management: description of high-cost addictions treatment clients. J Subst Abuse Treat 2013;45:280-6.

4. The economic impact of illicit drug use on American society. Washington (DC): US Department of Justice, National Drug Intelligence Center; 2011.

5. Rosenthal ES, Karchmer AW, Theisen-Toupal J, et al. Suboptimal addiction interventions for patients hospitalized with injection drug use-associated infective endocarditis. Am J Med 2016;129:481-5.

6. Baxter LE Sr, Campbell A, Deshields M, et al. Safe methadone induction and stabilization: report of an expert panel. J Addict Med 2013;7:377-86.

7. Martin JA, Campbell A, Killip T, et al. QT interval screening in methadone maintenance treatment: report of a SAMHSA expert panel. J Addict Dis 2011;30:283-306.

8. Lai SH, Yao YJ, Lo DS. A survey of buprenorphine related deaths in Singapore. Forensic Sci Int 2006;162:80-6.

9. Kintz P. Deaths involving buprenorphine: a compendium of French cases. Forensic Sci Int 2001;121:65-9.

10. Tetrault JM, Fiellin DA. Current and potential pharmacological treatment options for maintenance therapy in opioid-dependent individuals. Drugs 2012;72:217-28.

11. Dean M. Opioids in renal failure and dialysis patients. J Pain Symptom Manage 2004;28:497-504.

12. Methadone Program. Ottawa: Health Canada; 2016. Available: www.hc-sc.gc.ca/hc-ps/substancontrol/exemptions/methadoneeng.php (accessed 2016 June 9).

13. Handford C, Kahan A, Srivastava A, et al. Buprenorphine/ naloxone for opioid dependence: clinical practice guideline. 145 p. Ottawa: Centre for Addiction and Mental Health; 2011.

14. Oviedo-Joekes E, Guh D, Brissette S, et al. Hydromorphone compared with diacetylmorphine for long-term opioid dependence: a randomized clinical trial. JAMA Psychiatry 2016;73:447-55.

15. Holt SR, Ramos J, Harma MA, et al. Prevalence of unhealthy substance use on teaching and hospitalist medical services: implications for education. Am J Addict 2012;21:111-9.

16. Kouimtsidis C, Reynolds M, Hunt M, et al. Substance use in the general hospital. Addict Behav 2003;28:483-99.

17. Manchikanti L, Atluri S, Trescot AM, et al. Monitoring opioid adherence in chronic pain patients: tools, techniques, and utility. Pain Physician 2008;11(Suppl):S155-80.

18. Detoxification and substance abuse treatment. Treatment Improvement Protocol (TIP) Series No. 45. DHHS Publication No. SMA 15-4131. Rockville (MD): Center for Substance Abuse Treatment; 2006.

19. Kosten TR, O'Connor PG. Management of drug and alcohol withdrawal. N Engl J Med 2003;348:1786-95.

20. Kampman K, Jarvis M. American Society of Addiction Medicine (ASAM) national practice guideline for the use of medications in the treatment of addiction involving opioid use. J Addict Med 2015;9:358-67.

21. Meader N. A comparison of methadone, buprenorphine and alpha(2) adrenergic agonists for opioid detoxification: a mixed treatment comparison meta-analysis. Drug Alcohol Depend 2010;108:110-4.

22. McNicholas L. Clinical guidelines for the use of buprenorphine in the treatment of opioid addiction. Treatment Improvement Protocol (TIP) Series No. 40. DHHS Publication No. SMA 04-3939. Rockville (MD): Substance Abuse and Mental Health Services Administration; 2004.

23. Gowing L, Farrell M, Ali R, et al. Alpha ${ }_{2}$-adrenergic agonists for the management of opioid withdrawal. Cochrane Database Syst Rev 2016;(5):CD002024.

24. Jungquist CR, Karan S, Perlis ML. Risk factors for opioid-induced excessive respiratory depression. Pain Manag Nurs 2011;12:180-7.

25. Boyer EW. Management of opioid analgesic overdose. $N$ Engl $J$ Med 2012:367:146-55.

26. Alford DP, Compton P, Samet JH. Acute pain management for patients receiving maintenance methadone or buprenorphine therapy. Ann Intern Med 2006;144:127-34.

27. Gordon DB, Dahl JL, Miaskowski C, et al. American Pain Society recommendations for improving the quality of acute 
and cancer pain management: American Pain Society Quality of Care Task Force. Arch Intern Med 2005;165:1574-80.

28. Ti L, Voon P, Dobrer S, et al. Denial of pain medication by health care providers predicts in-hospital illicit drug use among individuals who use illicit drugs. Pain Res Manag 2015;20:84-8.

29. McNeil R, Small W, Wood E, et al. Hospitals as a 'risk environment': an ethno-epidemiological study of voluntary and involuntary discharge from hospital against medical advice among people who inject drugs. Soc Sci Med 2014;105:59-66.

30. Mehta V, Langford RM. Acute pain management for opioid dependent patients. Anaesthesia 2006;61:269-76.

31. Dowell D, Haegerich TM, Chou R. CDC guideline for prescribing opioids for chronic pain - United States, 2016. JAMA 2016;315:1624-45.

32. Rosenblatt AB, Mekhail NA. Management of pain in addicted/ illicit and legal substance abusing patients. Pain Pract 2005;5:2-10

33. Peng PW, Tumber PS, Gourlay D. Review article: perioperative pain management of patients on methadone therapy. Can J Anaesth 2005;52:513-23.

34. Merrill JO, Rhodes LA, Deyo RA, et al. Mutual mistrust in the medical care of drug users: the keys to the "narc" cabinet J Gen Intern Med 2002;17:327-33.

35. Berg KM, Arnsten JH, Sacajiu G, et al. Providers' experiences treating chronic pain among opioid-dependent drug users. J Gen Intern Med 2009;24:482-8.

36. Karasz A, Zallman L, Berg K, et al. The experience of chronic severe pain in patients undergoing methadone maintenance treatment. J Pain Symptom Manage 2004;28:517-25.

37. Scimeca MM, Savage SR, Portenoy R, et al. Treatment of pain in methadone-maintained patients. Mt Sinai J Med 2000;67:412-22.

38. Savage SR, Kirsh KL, Passik SD. Challenges in using opioids to treat pain in persons with substance use disorders. Addict $\mathrm{Sci}$ Clin Pract 2008;4:4-25.

39. Guidelines for the psychosocially assisted pharmacological treatment of opioid dependence. Geneva (Switzerland): World Health Organization; 2009.

40. Dunn KE, Brooner RK, Clark MR. Severity and interference of chronic pain in methadone-maintained outpatients. Pain Med 2014;15:1540-8.

41. Bryson EO. The perioperative management of patients maintained on medications used to manage opioid addiction. Curr Opin Anaesthesiol 2014;27:359-64.

42. Myers DP, Augustyniak M, Molea J. Buprenorphine for pain management physicians: A dilemma or a therapeutic alternative? Tech Reg Anesth Pain Manage 2005;9:216-20.

43. Gevirtz C, Frost EA, Bryson EO. Perioperative implications of buprenorphine maintenance treatment for opioid addiction. Int Anesthesiol Clin 2011;49:147-55.
44. O'Connor PG, Fiellin DA. Pharmacologic treatment of heroindependent patients. Ann Intern Med 2000;133:40-54

45. Yong TY, Fok JS, Hakendorf P, et al. Characteristics and outcomes of discharges against medical advice among hospitalised patients. Intern Med J 2013;43:798-802.

46. Seaborn Moyse H, Osmun WE. Discharges against medical advice: a community hospital's experience. Can J Rural Med 2004;9:148-53.

47. Choi M, Kim H, Qian H, et al. Readmission rates of patients discharged against medical advice: a matched cohort study. PLoS One 2011;6:e24459.

48. Hwang SW, Li J, Gupta R, et al. What happens to patients who leave hospital against medical advice? CMAJ 2003;168:417-20

49. Onukwugha E, Saunders E, Mullins CD, et al. Reasons for discharges against medical advice: a qualitative study. Qual Saf Health Care 2010;19:420-4.

50. Ti L, Milloy MJ, Buxton J, et al. Factors associated with leaving hospital against medical advice among people who use illicit drugs in Vancouver, Canada. PLoS One 2015;10:e0141594.

51. Suzuki J, DeVido J, Kalra I, et al. Initiating buprenorphine treatment for hospitalized patients with opioid dependence: a case series. Am J Addict DOI: 10.1002/AJAD.12161.x

52. Morozova O, Dvoryak S, Altice FL. Methadone treatment improves tuberculosis treatment among hospitalized opioid dependent patients in Ukraine. Int J Drug Policy 2013;24:e91-8.

53. Liebschutz JM, Crooks D, Herman D, et al. Buprenorphine treatment for hospitalized, opioid-dependent patients: a randomized clinical trial. JAMA Intern Med 2014;174:1369-76.

54. Shanahan CW, Beers D, Alford DP, et al. A transitional opioid program to engage hospitalized drug users. J Gen Intern Med 2010;25:803-8.

55. Chan AC, Palepu A, Guh DP, et al. HIV-positive injection drug users who leave the hospital against medical advice: the mitigating role of methadone and social support. J Acquir Immune Defic Syndr 2004:35:56-9.

56. Huxtable CA, Roberts LJ, Somogyi AA, et al. Acute pain management in opioid-tolerant patients: a growing challenge. Anaesth Intensive Care 2011;39:804-23.

Affiliation: Department of General Internal Medicine, Yale University School of Medicine, New Haven, Conn.

Contributors: All of the authors made substantial contributions to the conception and design of the work and to drafting the manuscript and revising it critically for important intellectual content, provided final approval of the version to be published and agreed to act as guarantors of the work. 